\title{
Cidadão Sensor e Cidade Inteligente: Análise dos Aplicativos Móveis da Bahia
}

\section{Citizen as Sensor and Smart City: Bahia's Mobile Apps Analysis}

André Lemos

Universidade Federal da Bahia, Salvador, BA, Brasil Orcid: 0000-0001-9291-6494

<almlemos@gmail.com>

\author{
Nayra Veras de Araújo
}

Universidade Federal da Bahia, Salvador, BA, Brasil Orcid: 0000-0002-1582-1704

<nayraveras@hotmail.com>

\section{Como citar este artigo (How to cite this article): \\ LEMOS, André; ARAÚJO, Nayra Veras. Cidadão Sensor e Cidade Inteligente: Análise dos Aplicativos Móveis da Bahia. Revista Famecos, Porto Alegre, v. 25, n. 3, p. 1-19, setembro, outubro, novembro e dezembro de 2018: ID28708. DOI: http://dx.doi.org/10.15448/1980-3729.2018.3.28708.}

\section{RESUMO}

O presente artigo apresenta um mapeamento dos aplicativos para dispositivos móveis disponíveis aos cidadãos pelo poder executivo do governo do Estado da Bahia e da Prefeitura Municipal de Salvador. Buscase analisar suas funcionalidades, serviços, setores e atividades. Está em jogo a ideia de um "cidadão inteligente" como um fornecedor de informação aos poderes públicos. Os resultados revelam uma visão tecnocrática, centrada mais na oferta de serviços no ambiente on-line e na gestão dos problemas do que na comunicação ampla entre os cidadãos. Os dados da pesquisa são apresentados em três aspectos: contexto do governo móvel e das smart cities; as características dos "cidadão-sensor" e descrição e análise dos aplicativos oficiais da Prefeitura e do Governo da Bahia.

\section{ABSTRACT}

The paper aims to show an exploratory research about the use of mobile applications by the State of Bahia and the Municipality of Salvador, Bahia's capital, Brazil. It seeks to identify how they are materially configured, their functionality, and structures. The results are subdivided into three main aspects: 1) An overview of services and functions; 2) Planning: how the applications are managed and understanding by the government and the city hall and 3) Monitoring: how the governments control the use of the mobile applications. At stake is an idea of a smart citizen, as an information provider to the public authorities.

Keywords: Mobile Applications. Smart City. Smart Citizen

Palavras-chave: Aplicativos Móveis. Cidade Inteligente. Cidadão Inteligente

\section{INTRODUÇÃO}

Soluções de governo usando tecnologias móveis (m-government) constituem hoje importantes ferramentas de comunicação pública, compreendidas como uma ação articulada e integrada entre setores estatais e governamentais cujo foco é o estreitamento das relações com o cidadão (Zémor, 2015; Duarte, 2009; Haswani, 2013). Nesse cenário, os aplicativos podem ser excelentes mediadores para incentivar processos comunicacionais multilaterais que primem não só pela comunicação governo-cidadão, mas também na relação cidadão-governo e cidadão-cidadão. 
A utilização de aplicativos para auxiliar o governo é uma realidade, embora ainda incipiente ${ }^{1}$. Na gestão pública da Bahia, tanto municipal quanto estadual, se configura como uma prática recente, iniciada há aproximadamente quatro anos, mas que vem se intensificando, justificando a necessidade de uma reflexão mais cuidadosa².

Embora verifique-se argumentos que defendam o uso dos aplicativos como potencializadores da comunicação entre Estado e sociedade (Bouskela,2016; Höffken, Streich, 2013), ainda não há padrões definidos sobre as melhores práticas no âmbito do governo, nem pesquisas mais elaboradas sobre essas ferramentas. Não há muita clareza sobre como elas têm sido pensadas no âmbito da administração pública. O seu desenvolvimento vem carregado de discursos sobre a melhoria da oferta de serviços e da comunicação com o cidadão. Há uma dimensão utópica e tecnocrática, apontando para uma mais eficiente da gestão institucional.

Partindo dessa constatação, o presente trabalho ${ }^{3}$ tem como objetivo mapear e descrever as funcionalidades, serviços, setores e atividades dos aplicativos móveis oficiais do governo do Estado da Bahia e da Prefeitura Municipal de Salvador. Na base da adoção desses aplicativos está a visão do usuário como um "cidadão sensor" que fornece dados aos gestores públicos. Criar aplicativos é, na maioria dos casos, produzir mecanismos de extração de dados do cidadão, seja em suas ações cotidianas mais banais, como pegar um ônibus, seja em uma participação mais ativa na vida pública, como participar com opiniões na definição do orçamento municipal, ou enviando sugestões e queixas sobre os problemas de infraestrutura urbana.

O texto encontra-se estruturado em três partes, além dessa introdução e uma conclusão: 1. O contexto do governo móvel e das smart cities; 2. As características dos "cidadão-sensor"; 3. A descrição e análise dos aplicativos oficiais da Prefeitura e do Governo da Bahia:

\footnotetext{
1 No Brasil, pesquisa realizada em 2015 pelo Centro Regional de Estudos para o Desenvolvimento da Sociedade da Informação (Cetic.br) revela que apenas $4 \%$ das prefeituras brasileiras oferecem serviços via aplicativos móveis.

2 Ver o eixo "cidade inteligente" do programa "Salvador 360" da Prefeitura de Salvador. http://360. salvador.ba.gov.br

3 Esse artigo faz parte da pesquisa "A Comunicação das Coisas. Internet das Coisas, Big Data e Smart Cities" (CNPQ/303461/2013-6) realizada no âmbito do Lab404 - Laboratório de Pesquisa em Mídia Digital, Redes e Espaço do Programa de Pós-Graduação em Comunicação e Cultura Contemporâneas da Faculdade de Comunicação da UFBA.
} 


\section{M-GOVERNMENT E SMART CITIES}

Empresas públicas e privadas têm se esforçado para apresentar soluções rápidas e eficientes no âmbito do Estado. Uma dessas soluções se dá pelo uso de tecnologias móveis e é denominada de "governo móvel" (m-government), um processo natural de evolução de práticas de governo eletrônico (e-government). Esta solução está vinculada à modernização da administração pública por meio do uso de tecnologias de informação e comunicação (TIC), à melhoria da eficiência dos processos operacionais e administrativos e dos serviços públicos oferecidos aos cidadãos (Diniz, 2009).

O m-government refere-se a um conjunto de estratégias conduzidas por setores públicos usando as tecnologias e dispositivos de comunicação móveis (computação nas nuvens, internet sem fio, smartphones e tablets) ampliando os canais de serviços e as formas de acesso a informações (Wendy Li, 2016). Um dos instrumentos mais importantes são os aplicativos governamentais utilizados por telefones celulares e tablets. A otimização de custos, a melhoria de comunicação e coordenação de dados, o acesso a grupos marginalizados (idosos, pessoas com deficiência, cidadãos de áreas rurais), a personalização de serviços, a mobilidade e a onipresença dos dispositivos seriam facilitadores dessas tecnologias para a conexão entre os cidadãos com o governo (OECD, 2011). No Brasil, o Departamento de Governo Eletrônico foi criado em 2004 e em 2013 o governo federal criou uma central de aplicativos móveis que reúne informações sobre os aplicativos desenvolvidos por órgãos governamentais do executivo federal. (Lima, 2017).

A discussão sobre m-government está relacionada ao debate sobre "cidades inteligentes" (smart cities). Cidades inteligentes são projetos de iniciativas públicas e/ou privadas, marcados pelo uso das TIC tendo como objetivo o desenvolvimento sustentado e a melhoraria da qualidade de vida dos cidadãos, ampliando as formas de participação e interação com poder público (Albino e outros, 2015; Bouskela, M. e Outros, 2016; Cavada e Outros, 2014; Deakin e Al Waer, 2012, De Waal, 2011; Galdón, 2013; Greenfield, 2013, Gurstein, 2014, Hollands, 2008; Kitchin, 2015; Kresin, 2013, Nam e Pardo, 2014, Schaffers e Outros, 2011, Townsend, 2013).

Zandbergen (2017) ressalta que o objetivo principal dos projetos de cidade inteligente é a maior eficiência da administração pública, da comunicação e da descentralização política. O adjetivo "inteligência" é entendido como o processamento automático da informação e a análise de um grande volume de dados. São muitas promessas e ainda poucas experiências que possam servir de base para uma afirmação segura sobre as consequências de implementação 
desses projetos. Mas é certo que todas as grandes cidades do mundo, em maior ou menor escala, estão aderindo a esse movimento.

Com o sucesso mundial do uso dos smartphones, os aplicativos tornamse um importante aliado do governo móvel e na implantação de cidades inteligentes. Höffken e Streich (2013) argumentam que essas tecnologias móveis abrem novos canais de comunicação entre os cidadãos e as instituições públicas e privadas. O cidadão oferece informação ao sistema e, ao mesmo tempo, recebe maior acesso aos serviços públicos. Os autores examinam as novas oportunidades abertas por celulares para a participação móvel no contexto dos processos de planejamento urbano, defendendo que estes abrem novos canais de comunicação, criam novas formas de coletar informações locais e criam oportunidades para participação cidadã.

De forma mandatória, ou pelo "faça você mesmo", os cidadãos estão no núcleo da ação, ao menos como fornecedores de informações às instituições governamentais e empresas privadas. Os aplicativos oficiais são uma das formas mais eficazes de colher informação voluntária. De acordo com Bouskela e outros (2016) além de facilitar a distribuição de alertas, serviços móveis e informações para habitantes, o uso de aplicativos permite ampliar a participação dos cidadãos, enviando dados e informação para os centros de gestão e controle da cidade. Na base desse discurso está a crença de que essas novas formas de interação social, agora móvel, colaborativa e interativa, via dispositivos móveis, revolucionam o uso da informação o engajamento político na atual sociedade informacional (Castells, 2003). Zandbergen (2017) adverte sobre esse ponto, afirmando que o discurso da colaboração urbana pode estar, na realidade, mascarando uma experiência de coleta de dados para interesses econômicos da uma política neoliberal. O papel do "cidadão-sensor" como fornecedor de informação, muitas delas pessoais e identificáveis, agrava as desconfianças e os temores.

\section{CIDADÃO - SENSOR}

Cidades inteligentes pressupõem "cidadãos inteligentes" (Kresin, 2013). Às vezes este atua como um ativista político, produzindo dados para questionamento do status quo, às vezes ele é apenas um fornecedor de informação. Pode-se pensar nesse cidadão inteligente como um "cidadãosensor" captando e produzindo informações em seu deslocamento pelo espaço urbano, seja na forma de dados primários, como em alguns exemplos de uso de Smart-Kits ${ }^{4}$, seja utilizando sites ou aplicativos governamentais para enviar

4 Trata-se de um kit com sensores para medir a composição do ar $\left(\mathrm{CO}\right.$ e $\left.\mathrm{NO}^{2}\right)$, temperatura, níveis de ruído, humidade e intensidade de luz. Os cidadãos podem usá-los colocando-os junto à janela de sua residência. O sistema foi adotado em Barcelona. 
informações, ou acessar serviços públicos. O termo aparece sobre as variantes: Citizen Sensing (Sheth, 2009), Human Sensor (Exner e outros, 2012), Citizens as sensors (Goodchild, 2007), ou Participatory Sensing (Burke, 2006).

$\mathrm{O}$ adjetivo faz referências a sensores digitais que captam informações fazendo leituras e traduções sobre grandezas do meio ambiente (temperatura, ruído, $\mathrm{CO} 2$ em dados digitais, por exemplo), enviando informações para a tomada de decisão em outros sistemas. De uma maneira ampla, todo cidadão deve ser um sensor dos problemas da polis. De uma forma mais limitada, ele apenas fornece informações para os poderes públicos, seja para melhorar sua eficiência burocrática, seja para solucionar efetivamente os problemas. Pode-se vincular o conceito ao uso dos aplicativos móveis.

Goodchild (2007) utilizou o termo Citizens as sensors para se referir ao engajamento generalizado de um grande número de cidadãos, muitas vezes com poucas qualificações formais. As práticas de colaboração do cidadão se referem a ações para informação geográfica voluntária (VGI). De forma similar, Sheth (2009) atribui o termo citizen sensing aos cidadãos que compartilham informações em dispositivos móveis e web 2.0. Eles criam o que o autor chama de Citizen Sensor Network (Redes de sensores cidadãos), interconectando pessoas que produzem informações sobre o espaço urbano (texto, áudio ou vídeo). Essa produção de informação integrada de dados, coletados via sensores maquínicos, com os fornecidos pelas redes de cidadãos fornece uma consciência situacional, ajudando a ação sobre o espaço urbano. Certamente os poderes públicos podem tirar proveito dessa"sensibilidade" cidadã, tanto para reagir com mais rapidez às demandas, como para ter mais informações locais e pessoais.

Podemos atribuir o conceito ao uso dos aplicativos atuais, principalmente quando se trata daqueles em que o cidadão é convidado a colaborar, fornecendo dados sobre problemas urbanos. Para Sheth (2009) as máquinas são boas no processamento simbólico, são pobres em conversão de informações sensoriais em símbolos ou palavras. Embora os sensores e os dispositivos atuais com alto processamento e machine learning possam recuperar esse atraso em relação à cognição humana, o que nos interessa aqui é ver como esse conceito pode ser usado para pensar o papel do cidadão no uso dos aplicativos.

Portanto, o conceito de "cidadão sensor" é interessante pois permite tensionar e vincular duas questões centrais: a cidadania e o processamento algorítmico. O primeiro nos permite pensar na ideia do cidadão como aquele que participa e colabora com a vida pública, agora, fortemente marcada pelo trânsito de informações digitais. O segundo, alerta para a coleta de dados voluntária e compulsória e a análise de dados que podem, por um lado, servir 
de estofo para as instituições criarem ações mais eficazes, mas também para ampliar regimes de vigilância e do controle.

Há dois tipos de ação do "cidadão-sensor": uma participativa, criando ferramentas que permitam usar os dados para ações de ativismo político (como os "smart citizens" de Amsterdã ou Barcelona) e outra mais corriqueira, usando aplicativos (oficiais ou não) para ação no espaço urbano, com consultas ou denúncias sobre os problemas de infraestrutura, e servir como porta de acesso à serviços. O uso dos aplicativos governamentais é o que caracteriza essa segunda forma de ação. O foco está mais centrado na ideia de sensor (que capta e oferece dados sem questionamento) do que na de cidadão (que age discursivamente). Nesse caso, ele é reduzido a um mero usuário.

$\mathrm{Na}$ atual fase da cultura digital o status de cidadania foi incorporado ao do direito à participação/inclusão social via dispositivos tecnológicos, posto que, a não adesão a utilização dessas ferramentas produz uma massa de excluídos digitais. $\mathrm{O}$ uso de ferramentas digitais para interagir com os poderes públicos muitas vezes é obrigatório e, mesmo quando é opção, a praticidade e a rapidez força os cidadãos a se tornarem "cidadãos-sensores". Os aplicativos são as ferramentas eficazes, pois são utilizados de forma voluntária. A centralidade do cidadão como fornecedor de dados aos gestores públicos pode, na verdade, induzir a novas formas de marginalização, exclusão, controle e vigilância. Como explica Caprotti (SCRUGGS, 2017, sem paginação),

Existe um risco real de reduzir os cidadãos e os seres humanos em geral a provedores de dados, por duas razões. Primeiro, nem todos os cidadãos fornecem dados. Então, se você não fornecer dados, ou se você não conectar esses fluxos de dados, o que acontece com você? Você se marginaliza do mundo do futuro próximo? Número dois, a governança através de dados e a governança através de métricas sempre carregam o potencial autoritário ${ }^{5}$

Há ainda mais dois problemas. O primeiro é o de só garantir a participação daqueles que têm acesso aos recursos tecnológicos. O segundo é que não se pode deixar de mencionar os fatores subjetivos como educação, motivação e interesse para a participação. Mais ainda, muitos dos que participam não têm

5 No original: There's a real risk of reducing citizens and human beings in general to providers of data, for two reasons. One, not all citizens provide data. So if you don't provide data, or if you don't plug into those data flows, what happens to you? Do you get marginalized from the world of the near future? Number two, governance through data and governance through metrics always carry with it authoritarian potential (Tradução dos autores). 
consciência do que acontece com os seus dados (para onde eles vão, quem os gerencia), ou do retorno que eles trazem para sociedade.

\section{Aplicativos oficiais da prefeitura e do governo da Bahia}

A pesquisa foi realizada em três etapas: exploratória (identificação dos aplicativos), coleta e análise de dados. A primeira foi desenvolvida entre setembro a outubro de 2016 e consistiu na verificação de informações no site da Prefeitura Municipal de Salvador, no Portal do Governo do Estado da Bahia, no Diário Oficial da Prefeitura de Salvador e em buscas na loja de aplicativos da Play Store ${ }^{6}$. A segunda foi realizada no mês de agosto de 2017 nas plataformas iOS e Android e identificou 21 aplicativos, sendo 12 do Governo e 9 da Prefeitura. A terceira analisou 16 aplicativos $^{7}$. A fim de identificar como esses aplicativos são gerenciados dentro da estrutura governamental, foram realizadas entrevistas com gestores públicos. O resultado da pesquisa é apresentado em três aspectos principais correspondentes a cada uma das fases da pesquisa: 1) Identificação dos Aplicativos e os Serviços Oferecidos; 2) Fluxos de Comunicação e 3) Monitoramento: manejo do uso dos aplicativos.

\section{Identificação dos aplicativos e os serviços oferecidos ${ }^{8}$}

O levantamento identificou 21 aplicativos associados às atividades da administração pública: Cittamobi, Noa Cidadão, Nota Salvador, SIMM Empresa, Coleta Seletiva, Mosquito Zero, Bike Salvador, Kim Recarga e Ouvindo Nosso Bairro, todos da Prefeitura. E SAC Bahia, Detran mobile, Agenda Cultural Bahia, TAG - Ouvidoria, Procon Mobile, Portal do Servidor, Caça Mosquito, Sine Bahia; Educar App, Diário oficial, Seia Mobile e Siga Mobile, do governo do Estado.

De acordo com a Organisation forEconomic Co-operation and Development (OECD), as práticas de m-government se estabelecem em quatro modelos de comunicação: governo - cidadãos (G2C); governo - governo (G2G), governo empresa (G2B) e governo - funcionários (G2E). Na Bahia, a presença de soluções móveis está distribuída em "públicos específicos"9 (empresas e servidor público)

6 Pesquisa da consultoria IDC revela que 95,5\% dos aparelhos comercializados entre julho e setembro de 2016 rodavam o sistema operacional Android no Brasil.

7 A análise não se concentrou em aplicativos utilizados internamente pela administração pública.

8 O panorama apresenta dados apenas do poder executivo. Não foram verificados o uso de aplicativos por outras instâncias do legislativo ou judiciário, como, por exemplo, Câmara Municipal, Tribunal de Contas, Tribunal de Justiça, Assembleia Legislativa entre outros.

9 Por exemplo: SIMM empresa (aplicativo "para empresa da Agência de Empregos da Prefeitura de Salvador"); Educar App (para o acompanhamento do desempenho escolar de estudantes do sistema público estadual) e Portal do Servidor (para servidores do governo do Estado). 
que, de acordo com a classificação da $\mathrm{OECD}$, inclui as práticas de G2G, G2B e G2E e "público geral" (qualquer cidadão) ou G2C.

Pelo volume de aplicativos, verifica-se que, tanto no governo da Bahia quanto na prefeitura de Salvador, o foco das ações é o público geral (G2C). No entanto, a oferta G2C nãogarante umfluxo efetivo decomunicaçãoe pode reforçar a posição do cidadão apenas como um fornecedor de dados. Esta constatação advém da observação sobre a diversidade de informações requisitadas dos usuários ao realizar o download e utilizar o aplicativo. Organizamos esses dados através das seguintes categorias de dados: do dispositivo, do usuário, de serviços e de redes sociais, os quais explicaremos a seguir.

\section{Dados do dispositivo}

Ao instalar o aplicativo, o usuário precisa autorizar o acesso a algumas informações do seu dispositivo. Esse procedimento pode indicar problemas de segurança e de privacidade. Apenas três aplicativos $(18,8 \%$,) não solicitam permissão. O acesso à localização e a fotos são as que mais aparecem (75\% e $68,8 \%$, respectivamente) - no Estado são as fotos e na Prefeitura a localização ${ }^{10}$. Nos dados agregados, em ordem decrescente, as outras solicitações ${ }^{11}$ são: câmera (31,3\%), identificação do dispositivo e informação da chamada (25\%), informação de conexão Wi-Fi (18,8\%), agenda (12,5\%), telefone (6,3\%) e histórico do dispositivo (6,7\%).

O acesso à localização do usuário garante a personalização dos serviços. Esse recurso está alinhado à temática da mobilidade, já que o sistema não se baseia em um endereço fixo (residencial ou de trabalho), mas no lugar de acesso. A OECD (2011) cita a localização como uma das maiores vantagens do governo móvel. Este recurso é utilizado, por exemplo, pelo aplicativo Cittamobi para informar o ponto de ônibus mais próximo do usuário, ou o Coleta Seletiva, fornecendo informação sobre postos mais próximos.

A requisição das fotos em grande parte dos aplicativos do Estado e da Prefeitura é em função da possibilidade de o cidadão enviar imagens sobre os problemas quotidianos. Isso poderia apontar para uma ação mais atenta do estado e/ou do município para uma comunicação de mão dupla. Não é o caso.

10 Nos aplicativos do governo do Estado temos fotos (75\%), localização (62,5\%), informações de conexão Wi-Fi (37,5\%), câmera (37,5\%), agenda (12,5\%) e informações de chamada (12,5\%). Em relação à Prefeitura: localização (87,5\%), fotos (62,5\%), acesso a informações de conexão Wi-Fi (37,5\%), câmera (25\%). O acesso à agenda, informações de chamada (12,5\%), identificação do dispositivo e histórico do dispositivo aparecem em menor proporção, cada um com 12,5\% respectivamente.

11 Os sistemas operacionais iOS e Android apresentam funcionalidades diferentes. Enquanto no Android a permissão deve ser realizada antes de realizar o download, no iOS a permissão só é solicitada no momento de acessar determinadas funcionalidades do aplicativo. 
A requisição das fotos aponta para um reforço da ideia do cidadão fornecedor de informação sem um retorno equivalente por parte do governo, sendo mais uma comunicação Cidadão para Governos (C2G) do que G2C.

Se o acesso à localização e às imagens do dispositivo é um aspecto que poderia garantir a melhoria na prestação de serviços, eles apresentam ameaças à segurança e à privacidade, já que envolvem a coleta de dados pessoais. Muitos dos aplicativos são desenvolvidos por empresas privadas e isso pode levar a uma distribuição desses dados para finalidades comerciais. Dos nove aplicativos utilizados pela Prefeitura, cinco são de desenvolvedores privados e no Governo apenas um $^{12}$.

\section{Dados do usuário}

Para acessar alguns aplicativos, total ou parcialmente, é necessário o cadastramento com login e senha. Este pode ser feito no próprio aplicativo ou por redes sociais (Facebook e Google). Em quase metade dos aplicativos (43,8\%) é possível ter acesso sem cadastramento (um da Prefeitura e seis do Governo ${ }^{13}$ ). Sem essa exigência o acesso é mais fácil, mas, por outro lado, perde-se em personalização no registro e no acompanhamento das informações através de um histórico de solicitações. Em 25\% dos aplicativos o login é necessário apenas para acesso a algumas funcionalidades. Em 31,3\% ele é necessário para acesso total. Para os que solicitam cadastro, as principais informações coletadas são: E-mail (62,5\%), Nome (56,3\%), telefone (56,3\%), senha $(43,8 \%)$, CPF (37,5\%), endereço (18,8\%). Outras informações aparecem como menor representatividade, como data de nascimento, sexo, estado, país. Nos aplicativos da Prefeitura são solicitadas as seguintes informações: e-mail (87,5\%), nome $(87,5 \%)$, telefone (75\%), senha (50\%), CPF $(50 \%)$, endereço $(37,5 \%)$. Para os do Governo são: nome (25\%), e-mail (50\%), senha (37,5\%), telefone (25\%).

\section{Dados de redes sociais}

O login aos aplicativos é feito, em algumas situações, via redes sociais. Além disso, 25\% dos aplicativos apresentam acesso ou compartilhamento integrado com Facebook, Twitter e Instagram. Dos aplicativos conectados com as redes sociais, 13,3\% direcionam para a página oficial do órgão/secretaria

12 Públicos são os aplicativos desenvolvidos por um órgão público (estadual, municipal ou federal). Privados são aplicativos desenvolvidos por empresas privadas (através de contrato, licitação, cessão etc.). 13 O percentual mais significativo de exigência de cadastro por parte da Prefeitura indica que esta tem acesso a uma planilha de dados mais detalhada levando a uma melhor orientação para a oferta de serviços públicos. 
a que está vinculado, 6,7\% para a página oficial do desenvolvedor e 6,1\% em páginas do próprio aplicativo.

\section{Divulgação de informações e a coleta de dados sobre serviços}

Foram organizadas nessa categoria as informações oferecidas aos cidadãos e a contrapartida sobre os dados pessoais requisitados para prover o Estado de informações sobre a melhoria dos serviços públicos. Foi observado que os serviços se distribuem em três funções: 1) fornecer informações sobre serviços públicos (normas, procedimentos, endereços, notícias), 2) solucionar problemas remotamente (emitir contracheques, imprimir boleto, agendar atendimentos) e, 3) Coletar dados sobre o ambiente urbano e gestão pública através da colaboração voluntária dos cidadãos. Com os dados agregados do Estado e Prefeitura, metade dos aplicativos oferecem informações sobre serviços públicos, sejam gerais (endereços e agendas de eventos), ou dos procedimentos a serem adotados para acesso (emissão de documento, solicitação de segurodesemprego, endereços de hospitais e postos de recolhimento de material reciclável, informação sobre agenda de cursos e eventos culturais).

O acesso às notícias foi verificado em $26,7 \%$, seja no próprio aplicativo (20\%), seja direcionando para uma página web (6,7\%). Os principais conteúdos são relativos à secretaria ou órgão a que estão vinculados; a informações gerais sobre a prefeitura ou governo do Estado e parceiros. Alguns oferecem, ainda, acesso a relatórios sobre a gestão pública (como prestação de contas e licitação, por exemplo), política de privacidade, termos de uso e agenda de eventos.

Mais de um terço $(37,5 \%)$ dos aplicativos funcionam como canal para solicitar serviços online e resolver problemas remotamente (50\% Governo, 50\% Prefeitura). Já o registro de reclamação, denúncias e ocorrências estão presentes em 32,5\% dos aplicativos. Elas guardam relação com o poder de fiscalização do cidadão, ampliando as formas de comunicação pública. Essa funcionalidade é encontrada nos aplicativos Noa Cidadão, Mosquito Zero, Cittamobi, TAG ouvidoria, Caça Mosquito e Procon-BA. Alguns aplicativos fornecem um número de protocolo, através do qual o cidadão pode acompanhar o andamento da solicitação (Noa Cidadão, Cittamobi e Procon). O Mosquito Zero e Caça Mosquito não fornece protocolo, mas emite um feedback automático.

Metade dos aplicativos do governo do Estado fornece informações sobre endereço e orientações sobre serviço. Em 37,5\% há canais para solicitar 
serviços online ${ }^{14}$, em $25 \%$ o serviço de registro de ocorrências ou denúncias, em $25 \%$ notícias sobre o órgão e em 12,5\% a agenda dos serviços públicos. Nos aplicativos da Prefeitura observa-se que 62,5\% deles disponibilizam informações de endereços e orientação sobre serviços, sendo 37,5\% serviços online, $25 \%$ registro de ocorrências/reclamação, 12,5\% produção colaborativa de informações sobre o ambiente urbano e 12,5\% consulta.

\section{Fluxos de Comunicação}

Classificamos os modelos de comunicação em fluxo unidirecional, bidirecional e multidirecional. O primeiro está em metade dos aplicativos da prefeitura e do Estado. Este é um modelo de comunicação no qual apenas um dos polos é ativo, seguindo na direção cidadão-governo (primeiro tipo) ou governo-cidadão (segundo tipo). São exemplos desses tipos de aplicativos os estaduais: Diário oficial, Detran, Sine Bahia e SAC Bahia; e os da prefeitura: Coleta Seletiva, Ouvindo Nosso Bairro, Bike Cidadão e Kim recarga.

O fluxo bilateral está em 37,5\% dos aplicativos, tanto da Prefeitura, quanto do Estado. Este modelo revela uma possibilidade de "conversa" entre a entidade pública e o cidadão, materializada em canais tais como: fale conosco, comentários, e-mails, ou postagens pelo aplicativo. A existência de tais mecanismos, entretanto, não nos permite afirmar de um fluxo de comunicação efetivo, pois não houve um monitoramento quanto ao atendimento das demandas apresentadas. Apresentam fluxo bilateral os aplicativos da prefeitura Cittamobi, Noa Cidadão e Nota Salvador; e do governo Tag Ouvidoria, Procon Mobile e Agenda Cultural.

No fluxo multidirecional, a informação é direcionada para um órgão público responsável por gerenciar a informação, mas é divulgada em um ambiente aberto em que os cidadãos têm acesso às informações produzidas por outros cidadãos. Este tipo de comunicação está presente em apenas dois aplicativos (Caça-mosquito e Mosquito Zero). É importante ressaltar que mesmo em canais de votação eletrônica, que se pautam num sistema de consulta popular, como identificado no aplicativo Ouvindo Nosso Bairro, o usuário pode votar, mas não acompanhar o andamento das votações.

Poucos aplicativos são colaborativos (comunicação tipo cidadão para cidadão - (2C). As possibilidades de produção e comunicação de conteúdos próprios por parte dos cidadãos se dão através de textos com comentários

14 Como exemplo temos o SAC Bahia (o cidadão pode solicitar o agendamento de serviços para atendimento presencial), o Detran-BA (acesso a serviços como a situação da $\mathrm{CNH}$, histórico de multas e geração de taxas), o Sine Bahia (o cidadão pode consultar a situação do seu seguro desemprego ou a empresa pode enviar demanda de cursos de qualificação profissional). 
livres (50\%), seleção de opções em um menu pré-definido (31,3\%), postagem de fotografias (25\%), que servem com o rastro de índice comprobatório (Noa Cidadão e do Caça Mosquito) e informações geolocalizadas (18,8\%). Não foi identificada a possibilidade de compartilhar ou postar vídeos. Em relação especificamente ao Governo, verifica-se uma abertura maior para produção de conteúdo através de comentários textuais $(62,5 \%)$. No que se refere à Prefeitura esse percentual é de apenas 37,5\%.

\section{Entrevistas}

Através das entrevistas buscamos entender como os aplicativos são pensados dentro da estrutura governamental. Estado e Prefeitura têm órgãos de gestão das TIC, respectivamente a PRODEB (Companhia de Processamento de Dados do Estado da Bahia) e a COGEL (Companhia de Governança Eletrônica do Salvador $)^{15}$. No entanto, nenhuma das duas coordena o desenvolvimento dos aplicativos de forma integrada. No Governo, o desenvolvimento de aplicativos se dá mais no âmbito interno, enquanto na prefeitura por desenvolvedores externos.

De acordo com o diretor técnico da COGEL, cada aplicativo é idealizado de acordo com o interesse de cada órgão ou secretaria. Algumas secretarias do município solicitam auxílio da COGEL, mas há casos em que o desenvolvimento ou adoção do aplicativo ocorre sem qualquer interferência da companhia. Já no âmbito estatal, a existência de um órgão responsável pelo desenvolvimento de soluções tecnológicas facilita a organização e apresentação dos aplicativos. Os gestores de desenvolvimento da companhia afirmam que as secretarias têm autonomia. Como afirma o gestor de desenvolvimento da Prodeb, "não tem nenhum decreto, nenhuma ordem: elas podem contratar fora, podem desenvolver, podem hospedar até na loja do fornecedor."16

O planejamento e desenvolvimento de aplicativos, tanto no âmbito municipal comoestadual, podem acontecer dequatroformas:I.Estado/Prefeitura desenvolve através de seus órgãos internos; II. Estado ou Prefeitura desenvolve através de empresas terceirizadas; III. Empresas terceirizadas desenvolvem e vendem para Estado/Prefeitura; e IV. Empresas públicas desenvolvem e vendem para Estado/Prefeitura. Como exemplo de aplicativos de tipo I temos o Educar

15 A função da COGEL é "planejar, coordenar, executar e controlar as ações relacionadas à gestão da informação e da telecomunicação na Prefeitura Municipal do Salvador - PMS utilizando tecnologias avançadas para promover a oferta de serviços e informações por meio eletrônico, definir padrões para as formas eletrônicas de interação e implantar ferramentas de racionalização de procedimentos". Ver http://www.tecnologia.salvador.ba.gov.br/

16 Entrevista realizada em maio de 2017. 
App, o Detran mobile e o Tag ouvidoria. Como exemplo do tipo II temos o Noa Cidadão, planejado pela Superintendência de Trânsito de Salvador, mas desenvolvido por uma empresa terceirizada ${ }^{17}$. O Cittamobi pode ser citado como um aplicativo do tipo III. Ele foi desenvolvido por uma empresa externa e vendido/cedido para a Prefeitura ${ }^{18}$. No último tipo, encontramos aplicativos do Estado desenvolvidos e vendidos pela PRODEB. O órgão desenvolve aplicativos sob encomenda (Portal do Servidor, SAC Mobile e Procon), ou propõe soluções e vende para órgãos (Caça-Mosquito, por exemplo).

\section{Monitoramento}

Oquedenominamos demonitoramentofoiobservadosobredoisaspectos: as atualizações dos aplicativos e a estrutura interna de acompanhamento das demandas apresentadas via aplicativos.

A atualização revela a atenção das instâncias públicas para oferecer aplicativos melhores e mais seguros, corrigindo eventuais falhas e criando novas funcionalidades. Dos 21 aplicativos, obtivemos informação sobre atualização em 16 deles. Destes, nove foram atualizados em 2017, três no ano de 2016 (dois no primeiro semestre e um no segundo). Outros três estão sem atualizações recentes (dois em 2015 e um em 2014). Dos aplicativos do governo apenas três foram atualizados em 2017, dois em 2016 e três em 2015. 62,5\% dos aplicativos foram atualizados há mais de um ano, apontando para problemas de atualização ${ }^{19}$. No que tange a Prefeitura, verifica-se uma constância de atualizações menos espaçadas. $75 \%$ tiveram atualização em $2017^{20}$, um em 2015 e um em 2014.

Os aplicativos demandam graus variados de monitoramento (gerenciamento e acompanhamento das ações dos usuários) em razão da função que desempenham e dos serviços que oferecem. Aplicativos como Detran Mobile, por exemplo, não necessitam do mesmo tipo de monitoramento que um aplicativo como o Noa Cidadão, que se propõe a registrar ocorrências.

No âmbito do governo do Estado verifica-se que a maior parte dos aplicativos trata de versões de dispositivos móveis para páginas Web e,

17 Lançado em 11 de julho de 2016, o aplicativo foi desenvolvido pelo Grupo Recursos, empresa responsável pela estruturação do NOA.

18 A prefeitura realizou licitação concepção, desenvolvimento, fornecimento e implantação de um sistema integrado de gestão do transporte coletivo de ônibus. O Cittamobi é um subproduto oferecido pela empresa Cittati.

19 Esses dados não são suficientes para afirmarmos que a falta de atualização impacta no serviço oferecido pelo aplicativo.

20 O Kim Recarga e Ouvindo nosso Bairro foram criados em 2017. A data de atualização coincide com a data de lançamento. 
portanto, não há diferença entre o tipo de monitoramento dado ao aplicativo e ao ambiente Web (Agenda Bahia, Detran Mobile, SAC Mobile). Essa situação é diferente em aplicativos da Prefeitura como o Noa Cidadão e o Cittamobi. Em ambos os casos, o acompanhamento é feito através de uma central de comando e controle. No Cittamobi, ele é realizado pela SEMOB (Secretaria de Mobilidade Urbana), mais especificamente pelo Centro de Controle Operacional (CCO ${ }^{21}$.

\section{CONCLUSÃO}

Foi apresentado um primeiro panorama sobre os aplicativos oficiais utilizados pelo poder público executivo do Governo da Bahia e da Prefeitura de Salvador. Este trabalho é inédito no estado. Não há estudos similares. $O$ conceito de "cidadão sensor" permite tensionar a intenção desses aplicativos. Esses artefatos oferecem serviços úteis ao cidadão em troca de dados para os poderes. A pesquisa mostra que um "cidadão sensor"é convocado para participar de uma comunicação pública sem que saibamos exatamente seus contornos ${ }^{22}$.

A pesquisa permite apontar que a oferta de aplicativos no setor público municipal e estadual indica, por um lado, a busca por atualização tecnológica para colocar o município e o Estado na esteira do desenvolvimento de "cidades inteligentes". Por outro, visa ampliar a comunicação pública, seja para comunicações bidirecionais, seja para comunicações unidirecionais. Os aplicativos são certamente novas ferramentas para a comunicação pública.

Embora não haja elementos para afirmar a melhoria da comunicação entre o setor público e os cidadãos, há certamente uma intenção de avançar nesse sentido. Os dados mostraram que a comunicação entre cidadão e setor público se dá, predominantemente, de forma unidirecional. Ao cidadão é difícil fiscalizar e monitorar o setor público e saber da segurança e privacidade sobre seus dados. Não há um trabalho centralizado, nem no Estado nem no município, e isso pode levar a entender que se trata de um discurso centrado na inovação tecnológica e na modernização, na requisição de informação desse "cidadão sensor", mas as ferramentas ainda não estão suficientemente organizadas de modo a garantir uma ampliação da participação e uma comunicação mais multidirecional.

A falta de transparência sobre as ações demandadas através de aplicativos é um claro exemplo de que o cidadão não está no centro do processo. Mesmo

21 O CCO foi inaugurado na mesma data do lançamento do aplicativo, em 1 de janeiro de 2015. Ver http://www.mobilidade.salvador.ba.gov.br

22 Uma próxima etapa da pesquisa (já em andamento), busca colher, por meio de uma survey, as impressões dos usuários no uso desses aplicativos já que não se sabe exatamente quais os aplicativos mais usados, como ou quantas pessoas os usam. 
naquelas que se propõem a fomentar práticas colaborativas, não é possível acompanhar o desenvolvimento e andamento das ações. Esta constatação pode sugerir que o discurso da colaboração urbana pode estar mascarando uma experiência de coleta de dados para interesses econômicos (Zandbergen, 2017). Como apontado, alguns aplicativos são desenvolvidos por empresas externas a estrutura governamental e que, portanto, os dados disponibilizados pelos usuários podem atender a interesses privados.

Embora osaplicativos possam servistos comoferramentas de comunicação que coloquem o cidadão no centro do processo, o seu valor está certamente na coleta de dados gerados pelo sistema. A manutenção de bancos de dados com informações sobre os usuários e suas demandas é uma clara evidência dessa dimensão. Esses dados são fundamentais para a melhoria da gestão urbana, mas devem ser protegidos legalmente e tecnicamente, e os cidadãos devem ter consciência de que estão fornecendo dados sensíveis ao sistema. É necessário maior transparência sobre esses dados. Questões sobre vigilância, privacidade e proteção de dados pessoais parecem não estar no centro das atenções dos poderes públicos.

A Prefeitura de Salvador e o Governo do Estado da Bahia têm utilizado aplicativos para melhorar a oferta de serviços aos cidadãos, mas ambos mantêm uma visão tecnocrática, centrada mais na oferta de serviços no ambiente on-line e na gestão dos problemas do que na comunicação ampla entre os cidadãos, com o predomínio de uma comunicação unidirecional ou bidirecional, com poucas experiências de práticas colaborativas, criando ações de segundo tipo do "cidadão-sensor".

Certamente, os aplicativos para dispositivos móveis podem ser ferramentas importantes de interação entre governo e cidadão (m-government) e ampliar as práticas de comunicação pública, mas tanto na Prefeitura quanto no Governo o planejamento dessas ferramentas acontece de forma isolada, sem esforço integrado ou estratégico. Não há elementos para afirmar que eles façam parte de uma política de governo. Não há relatórios ou métricas de uso desses aplicativos fazendo com que não se saiba os reais impactos positivos ou negativos da sua implementação.

Por fim, apesar das críticas, acreditamos que iniciativas de participação móvel e de colaboração urbana são importantes para a comunicação pública e que essas experiências devem ser aperfeiçoadas no sentido de garantir maior visibilidade sobre a forma como são gerenciadas e para proporcionar formas de ação de primeiro tipo desse "cidadão-sensor". Apenas como fornecedor de dados, a ideia de "sensor" pode ser vista como incompatível com a ideia de cidadão, entendendo este como um ator ativo que atua e se mobiliza historicamente na 
conquista de seus direitos, e aquele como um ser passivo que apenas reage às mudanças do ambiente. Ações de primeiro tipo podem transformar essa visão do "sensor" como mais próxima desse cidadão alerta e que reage rápido aos problemas.

\section{REFERÊNCIAS}

ALBINO, V.; BERARDI, U.; DANGELICO, R. M. Smart Cities: Definitions, Dimensions, Performance, and Initiatives. Journal of Urban Technology, Londres, v. 22, n. 1, p. 3-21, Abril 2015. Disponível em: http://dx.doi.org/10.1080/10630732.201 4.942092. Acesso em: 22 nov. 2016.

BOUSKELA, M (e outros). Caminho para as Smart Cities: da gestão tradicional para a cidade inteligente. Banco Internacional de Desenvolvimento. BID, São Paulo, p. 33-144, 2016. Disponível em: https://goo.gl/kovU8r. Acesso em: 18 mar. 2017.

BURKE, J. A, ESTRIN, D., HANSEN, M., PARKER, A., RAMANATHAN, N., REDDY, S., \&, M. B. (2006). Participatory sensing. UCLA: Center for Embedded Network Sensing. Colorado (EUA), p. 1-6, maio 2006. Disponível em: https://escholarship.org/uc/ item/19h777qd. Acesso em: 20 jul. 2017.

CAVADA, M., ROGERS Y Dexter, H. Smart Cities: Contradicting Definitions and Unclear Measures. World Sustainability Forum 2014 - Conference Proceedings Paper, 2014.

CASTELLS, M. A Era da Informação: economia, sociedade e cultura, vol. 1, 9 ed. São Paulo: Paz e terra.

DE WAAL, M. The urban culture of sentient cities: from an internet of things to a public sphere of things. In: Sentient City. Ubiquitous Computing, Architecture, and the Future of Urban Space, MIT Press, Cambridge, 2011.

DEAKIN, M., AI WAER, H. From intelligent to smart cities, Routledge, Londres. 2012.

DINIZ e outros. O governo eletrônico no Brasil: perspectiva histórica a partir de um modelo estruturado de análise. Revista de Administração Pública - RAP. Rio de janeiro, n. 43, v. 1, p. 23-48, jan./fev. 2009.

DUARTE, J. (Org.). Comunicação Pública: estado, mercado, sociedade e interesse público. 2. ed. São Paulo: Atlas, 2009.

EXNER, J. P.; BERGNER, B. S.; ZEILE, P.; BROSCHART, D. Humansensorik in der räumlichen Planung. In: STROBL, J.; BLASCHKE, T.; GRIESEBNER, G. (Orgs.), Angewandte Geoinformatik 2012 - Beiträge zum 24ten AGITSymposium Salzburg: Wichmann Fachmedien, n. XXIV p. 690-699, 2012. Disponível em: https://goo. gl/iB1Njo. Acesso em: 22 set. 2017. 
GALDÓN, G. (Not so) smart cities?: The drivers, impact and risks of surveillanceenabled smart environments. Science and Public Policy, v. 40, n.6, p. 717-723, dezembro, 2013. Disponível em: https://goo.gl/pHrkJt. Acesso em: 18 set. 2017.

GOODCHILD, M. F. Citizens as Sensors: the World of Volunteered Geography. GeoJournal, v. 69, n. 4, p. 211-221, Novembro, 2007. Disponível em: https://goo.gl/ naj3gv. Acesso em: 18 set. 2017.

GREENFIELD, A. Against the smart city. Do Projects, Nueva York, 2013.

GURSTEIN, M. Open data: empowering the empowered or effective data use for everyone". First Monday, Chicago, v. 16, n. 2, janeiro, 2011. Disponível em: http:// www.uic.edu/htbin/cgiwrap/bin/ojs/index.php/fm/article/view/3316/2764. Acesso em: 21 ago. 2017.

GURSTEIN, M. Smart Cities vs. Smart Communities: Empowering Citizens not Market Economics. The Journal of Community Informatics, v. 10, n. 3, 2014.

HASWANI, M. F. Comunicação Pública: bases e abrangências. São Paulo: Saraiva, 2013.

HÖFFKEN, S., STREICH, B.: Mobile Participation: Citizen Engagement in Urban Planning via Smartphones. In: Silva, C. (Org.) Citizen E-Participation in Urban Governance: Crowdsourcing and Collaborative Creativity. Hershey, Pensilvânia (EUA). 199-225, jun., 2013. Information Science Reference, Hershey (2013). Disponível em: https://goo.gl/E18jTB. Acesso em: 10 jun. 2017.

HOLLANDS, R. Will the real smart city please stand up? City: Analysis of Urban Trends, Culture, Theory, Policy, Action, v. 12, n. 3, p. 303-320, 2008.

KITCHIN, R. Making sense of smart cities: addressing present shortcomings. Cambridge J Regions Econ Soc, v. 8, n. 1, 2015.

KRESIN, F. A manifesto for smart citizens. In: HEMMENT, D., TOWNSEND, A. (2013), Smart citizens, Future Everything, Manchester, 2013.

LEMOS, A. Smart Cities, internet of things and performative sensibility. Brief analysis on Glasgow, Curitiba and Bristol's Initiatives. P2P \& Inovação, Rio de Janeiro, v. 3, n. 2, p. 80-95, Mar./Set. 2017.

LIMA, C. C. B. de. M-Government: Limits and Possibilities for the Use of Mobile Apps as a Citizenship Tool in Brazil. Icegov '17: Proceedings of the 10th International Conference on Theory and Practice of Electronic Governance. New Delhi AA, India - March 07 - 09, 2017.

NAM, T; PARDO, T. A. Conceptualizing Smart City with Dimensions of Technology, People, and Institutions. The 12th Annual International Conference of Digital Government Research, 2011. Nova York, p. 282-291, 2011. Disponível em: 
https://www.researchgate.net/profile/Taewoo_Nam/publication/221585167_ Conceptualizing_smart_city_with_dimensions_of_technology_people_ and_institutions/links/0f31752f60bf009d2f000000/Conceptualizingsmart-city-with-dimensions-of-technology-people-and-institutions. pdf?origin=publication_detail Acesso em: 20 out. 2016.

OECD. M-Government: mobile technologies for responsive governments and connected societies, 2011.

SCHAFFERS, H., N. M. PALLOT, B. TROUSSE, M. NILSSON, Y A. OLIVEIRA. 2011. Smart Cities and the Future Internet: Towards Cooperation Frameworks for Open Innovation., In: DOMINGUE, John e outros (Orgs.) The Future Internet, Berlin, p. 431-446. Disponível em: https://link.springer.com/content/ pdf/10.1007\%2F978-3-642-20898-0.pdf. Acesso em: 21 set. 2017.

SCRUGGS, Gregory. The 'new urban citizen' and the dangers of the measurable city. Disponível em: http://www.urbangateway.org/news/'new-urban-citizen'-anddangers-mea. Acesso em: 25 ago. 2017.

SHETH, A. P. Citizen Sensing, Social Signals, and Enriching Human Experience. In: IEEE Internet Computing, Washington, v. 13, n. 4, p. 87-92, julho, 2009. Disponível em: http://corescholar.libraries.wright.edu/knoesis/728. Acesso em: 25 set. 2017.

TOWNSEND, A. Smart Cities: Big Data, Civic Hackers, and the Quest for a New Utopia. New York: W. W. Norton \& Company, 2013.

WENDY LI. Government and Mobile: A Gear Change? In: LANZA, Beatriz Barreto; CUNHA, Maria Alexandra Cunha (Orgs.). Human Social Interaction in the Age of Mobile Devices, 2016. p. 133-149.

ZANDBERGEN; D. "We Are Sensemakers": The (Anti-)politics of Smart City Cocreation. Public Culture 1, Durham, v. 29, n. 3, p. 539-562, set. 2017. Disponível em: https://doi.org/10.1215/08992363-3869596. Acesso em: 3 out. 2017.

ZÉMOR, P. La Communication Publique. PUF, COL, Que sais-je? Paris, 1995. 
Recebido em: 02/10/2017

Aceito em: 12/03/2018

Dados dos autores:

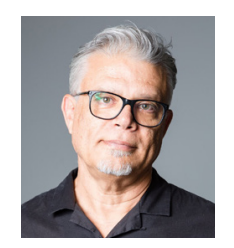

André Lemos | almlemos@gmail.com

Universidade Federal da Bahia

Professor Titular da Faculdade de Comunicação (FACOM) e do Programa de Pós-Graduação em

Comunicação e Cultura Contemporâneas da Universidade Federal da Bahia. Doutor em Sociologia pela

Université René Descartes, Paris V, Sorbonne (1995) e pós-doutor (visiting scholar) pela University of

Alberta e McGill University, Canadá (2007-2008) e National University of Ireland (Irlanda, 2015-2016).

Programa de Pós-Graduação em Comunicação e Cultura Contemporânea

R. Barão de Jeremoabo, S/N - Ondina.

40170-115 Salvador (BA) - Brasil

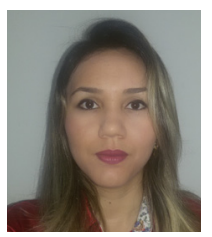

Nayra Veras de Araújo | nayraveras@hotmail.com

Doutoranda do programa de Pós-graduação em Comunicação e Cultura Contemporâneas da Universidade Federal da Bahia (UFBA) e mestre em Políticas Públicas. Pesquisadora do Laboratório de Pesquisa em Mídia Digital, Redes e Espaço (LAB404). Bolsista Capes.

Programa de Pós-Graduação em Comunicação e Cultura Contemporânea

Universidade Federal da Bahia

R. Barão de Jeremoabo, S/N - Ondina.

40170-115 Salvador (BA) - Brasil

Contribuições dos autores: Ambos, autor e autora, fizeram contribuições substanciais para concepção, desenvolvimento, redação e revisão crítica do trabalho; e aprovação final da versão para publicação. 